\title{
Development of a Comprehensive Driving Cycle for Construction Machinery Used for Energy Recovery System Evaluation: A Case Study of Medium Hydraulic Excavators
}

\author{
Peng Hu $\mathbb{C}^{\text {, }, 2}$ Jianxin Zhu, ${ }^{1,2}$ Jun Gong, ${ }^{3}$ Daqing Zhang, ${ }^{2}$ Changsheng Liu, \\ Yuming Zhao, ${ }^{2}$ and Yong Guo ${ }^{1}$ \\ ${ }^{1}$ State Key Laboratory of High Performance and Complex Manufacturing, Central South University, Changsha, China \\ ${ }^{2}$ The National Enterprise Research and Develop Center, Sunward Intelligence Equipment Co.,Ltd., Changsha, China \\ ${ }^{3}$ Engineering Research Center of Advanced Mining Equipment Ministry of Education, \\ Hunan University of Science and Technology, Xiangtan, China
}

Correspondence should be addressed to Peng Hu; hupeng@csu.edu.cn

Received 4 June 2020; Revised 9 February 2021; Accepted 15 February 2021; Published 23 February 2021

Academic Editor: Georgios Dounias

Copyright $\odot 2021$ Peng Hu et al. This is an open access article distributed under the Creative Commons Attribution License, which permits unrestricted use, distribution, and reproduction in any medium, provided the original work is properly cited.

Energy recovery and hybrid power technology are new directions in construction machinery energy-saving research. Thus far, however, no uniform standard method exists to evaluate the efficiency of novel energy-saving concept systems in early stages of development. Efficiency assessment is valuable and credible only by relying on driving cycles that are consistent with actual data. As representative products of construction machinery, hydraulic excavators are multifunctional, object-uncertain, and heavily influenced by operator habits. It is therefore challenging to develop standard excavator driving cycles. Aiming at the energy efficiency evaluation of new energy-saving products characterized by energy recovery and power system optimization, taking medium-sized hydraulic excavator as an example, this paper proposes an evaluation method of energy-saving efficiency and a classification construction method of comprehensive driving cycle of hydraulic excavator based on actual operating data with load demand power and boom recoverable power as combined variables. First, based on the analysis of general driving cycle variables for excavators with different energy-saving schemes, a load demand power model and boom recoverable power model based on machine sensing data are established. Second, 10 excavators were selected at different locations in southern China, and 30 days of real-world working data were recorded. Third, according to the periodic characteristics of working data, a method for dividing microcycle operation structure is proposed. The microcycle sample space based on the data conversion of working data is constructed and classified via the clustering algorithm. Finally, a comprehensive excavator driving cycle based on the classification results is constructed through the Markov method. Results show that the energy-saving efficiency of the three classification driving cycles was $17.45 \%, 13.60 \%$, and $11.88 \%$, respectively; the comprehensive energy-saving efficiency was $15.76 \%$. The deviations in maximum load demand power and maximum boom recoverable power between the constructed comprehensive cycle and the sample space were $7.81 \%$ and $8.61 \%$; the average deviation of characteristic parameters was $4.26 \%$. The comprehensive driving cycles can fairly reflect the general characteristics of real-world working conditions. The proposed construction method of comprehensive driving cycles based on sample space is therefore reliable and holds great promise for evaluating the energy-saving efficiency of new energy-saving concept systems.

\section{Introduction}

Resource shortages and environmental pollution are increasingly urgent global issues that have promoted the development of energy-saving and emission-reduction technologies [1-4]. As a typical form of construction machinery, hydraulic excavators (HEs) have characteristics of large usage, high energy consumption, and poor emissions. Energy recovery and hybrid power technology are important energy-saving techniques [5-8], but the implementation of 
both is highly dependent on specific driving cycles. Standard driving cycles can be used to evaluate pollution emissions and fuel consumption of new energy-saving systems [9].

In the vehicle industry, standard driving cycles have developed rapidly. Industry organization-led and government-led standards mainly include the US driving cycle, European driving cycle, and Japanese driving cycle [10]. The driving environment, road level, and driving habits in different regions are distinct; driving cycles often differ greatly from real-world driving characteristics of the local area, which causes test results to deviate substantially from actual working conditions [11]. Therefore, many researchers have analyzed the driving characteristics of local areas and constructed driving conditions consistent with specific cities. Lin and Niemeier [12] collected driving data from three regions in California and found that regional changes caused notable differences in acceleration, deceleration, uniform velocity, and idle states while driving. Andre et al. [9] tested the emission levels of 30 passenger cars using standard and targeted-development driving cycles. Findings indicated it was difficult to cover all models with a single driving cycle, which led the test results to be inconsistent with real working conditions. Seers et al. [13] developed a targeted driving cycle based on driving conditions and vehicle types, which was compared with the FTP75. Results showed that fuel consumption and emissions measured by the FTP75 were significantly lower than those evaluated using the targeted cycle. Although representative working conditions can be constructed according to local conditions to ensure that test results are consistent with actual working conditions, the establishment of a driving cycle will also influence test results [14].

Compared with the vehicle industry, research on standard driving cycles in the excavator field lags somewhat behind. There are three types of relevant literatures. The first is the factory test method for functional verification of excavator products. In BS ISO 10987-3-2017 Earth-moving machinery-Sustainability, fuel consumption data analysis guidelines are proposed. ISO 11152 "Test method for energy consumption of Earth-moving machinery" [15], which is under development, will standardize a specific energy consumption test method and determine typical working condition proportions for two types of machines (i.e., a wheel loader and HE). The Japan Construction Machinery Association has proposed Earth-moving machinery-Test methods for energy consumption-hydraulic excavators" (JCMASH020:2008) [16]; JCMAS H020 sets a comparative test procedure for HEs. Four working conditions are considered to outline ordinary machine use: trench digging, soil leveling, straight traveling (forward/back), and low idling standby. JCMAS H020 relates a machine's operational efficiency to specific fuel consumption values, and a calculation procedure for such values is defined for each working condition. The hydraulic excavator test method (GB/T 75862008) [17] developed in China has been standardized from measurements of the driver's control device and traveling speed along with test methods for braking performance.

The second type is the study of the load spectrum to improve machine design and fatigue life analysis. Yin et al.
[18] highlighted the need for fatigue life prediction of cracked components. Field monitoring of a cable shovel boom with a history of chronic fatigue cracking was conducted. A set of load spectra for the boom was established from gathered field data. Xiang et al. [19] proposed a load spectrum test method to reflect the actual operating conditions of an HE. Based on a test of the cross-section internal force, the cross-section was selected at a stress-free location of the bucket rod. Zhang and Zhai [20] tested a HE and collected corresponding load data under typical working conditions. Based on the measured data, the load segmentation of hydraulic pumps was standardized according to segmentation characteristics of the working conditions. Arsić et al. [21] presented the results of tests and analyses of complex dynamic loads performed on a bucket-wheel excavator SchRs 650/524 Krupp. They also assessed the service life of vital welded structures in a bucket-wheel excavator boom subjected to cyclic loading with a variable amplitude. Using experimental tests, they determined the operational strength and growth of a fatigue crack for a specific structural part.

The third type involves evaluating operating methods for automatic excavator control. Zarotti et al. [22] analyzed a standard test procedure consisting of four main working conditions to evaluate machine performance. A standard operating cycle was defined for each condition. Among them, trench digging was most important. Yoon et al. [23] studied the movement of three excavators under different spatial conditions. The spatial factors affecting loading operations were identified and investigated in terms of different machine movements. Kim and Chi [24] proposed a visionbased action recognition framework that considers sequential working patterns of earth-moving excavators for automated cycle time and productivity analysis. The sequential patterns of visual features and operation cycles were incorporated into an action recognition framework.

In summary, no uniform method is available to evaluate the energy efficiency of new energy-saving concept systems in early stages of development. Efficiency evaluations are valuable and reliable and focus on driving cycles consistent with real-world data. Compared with vehicles, it is challenging to develop standardized operating standards for HEs for several reasons. First, HEs are multifunctional work equipment, and working conditions are often diverse; thus, it can be challenging to reflect real-world operational characteristics under uniform standard conditions. Second, changes in the working object (e.g., soil and sand with different mechanical properties affecting excavator system performance) are highly uncertain and difficult to describe with uniform standard working conditions. Third, the driver's operating habits affect excavator performance. Drivers' habits are also random and difficult to describe with a unified model. HEs are multifunctional, object-uncertain, and highly affected by operator habits [25], which makes it difficult to develop standard driving cycles.

After the analysis of driving cycle variables, we collected long-term real-world working data from 10 medium-sized HEs in different regions of southern China. The microcycle structure and corresponding characteristic parameters of the 
excavators were defined, and a working database of the excavators was constructed. Based on principal component analysis of feature parameters, a clustering algorithm was used to classify various working conditions. According to the classification results, the Markov method was used to construct a comprehensive driving cycle. In line with aforementioned studies, the main contributions of this work are as follows: (1) a microcycle description method is proposed based on the time-power profile combining load demand and degree of energy recovery; (2) a construction method for the sample space of excavator microcycle based on the conversion of working data is proposed, and the standardization of multifunctional driving cycle of a realworld excavator is realized based on a clustering algorithm; (3) a random problem model is developed based on the Markov method to realize the description of random changes of the operation objects and driver's habits.

The remainder of this paper is organized as follows. The operating principle and power estimation model of medium HE systems are presented in Section 2. Section 3 outlines the microcycle structure and cluster analysis method for HEs. A comprehensive driving cycle is built based on a Markov chain and presented in Section 4, and conclusions are drawn in Section 5.

\section{Operating Principle and Power Estimation Model of Medium HE System}

2.1. Operating Principle. HEs mainly include five actuators: a boom, arm, bucket, rotary platform, and travel device (see Figure 1). Through a flexible combination of these five actuators, various work functions can be realized, including digging, hauling, dumping, swinging, and traveling. 20T-class mediumsized HEs are the most common tonnage. The HE studied in this paper is a $23 \mathrm{~T}$ tracked HE SWE230B produced by SUNWARD Intelligent Equipment Co., Ltd. The main parameters of the device have been outlined at [26]. The operating principle of the SWE230B power system is shown in Figure 2. A dual-pump negative flow system is used for power distribution between multiple actuators. The main control valve consists of a series and parallel connection of several valve blocks. The individual and combined drive of the two pumps can be achieved via the main control valve. Each valve block is pushed by the pilot pressure $p_{i}$ output via the corresponding hydraulic joystick to achieve throttling speed regulation. The main control valve center outlet is connected to the tank through orifices at the end of the main control valve. The pressure $\left(p_{s 1}\right.$ and $\left.p_{s 2}\right)$ established before the orifices can negatively affect the flow-matching characteristics of the hydraulic pump and actuator (large pressure indicates more pump flow is not being used by the actuator). Therefore, the pump displacement is reversely adjusted by the median pressure loss feedback signals $p_{s 1}$ and $p_{s 2}$ of the main control valve, thereby reducing pump flow loss.

2.2. Power Estimation Model. For vehicles, a velocity-time profile can be used to define the driving cycle. Because HEs comprise a type of working equipment, the velocity-time profile cannot reflect the actual excavator load. HEs also

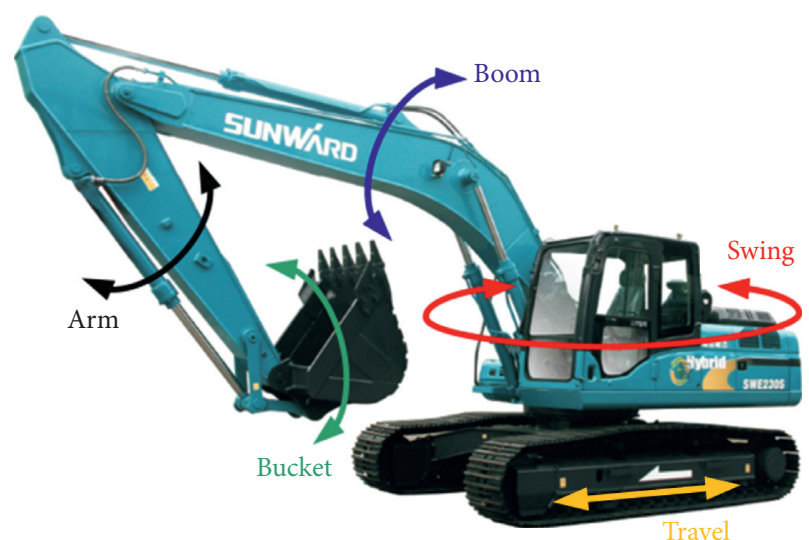

Figure 1: Hydraulic excavator actuator action diagram.

have multiple degrees of freedom; thus, defining a driving cycle with different actuator velocity combinations will result in excessive data. As the purpose of this study is to propose a driving cycle for energy efficiency assessment, the result of the interaction between the work object, system, and driver (i.e., the power-time profile) is used to define the excavator driving cycle. Such a driving cycle ignores differences in the system structure and can be used to compare excavators with greater adaptability. Considering the energy recovery and energy efficiency analysis of the drive system, there are two main relationships: load demand power to time $P_{L}(t)$ and boom recoverable power to time $P_{B}(t)$. The former is used to characterize power requirements of the excavator system for the prime mover (in this case, the diesel engine); the latter is used to characterize conversion of gravitational potential energy during the boom-lowering process (i.e., theoretical recoverable power).

However, these two parameters are difficult to measure for conventional excavator products. To obtain numerous data in the actual excavator working environment, these parameters must be estimated via a particular mathematical model based on an existing excavator sensing system. In the SWE230B system, the following variables are measured: main pump pressures $p_{1}$ and $p_{2}$; negative flow pressures $p_{s 1}$ and $p_{s 2}$; boom-lowering pilot pressure $p_{i}$; and engine speed $n_{E}$. To analyze the boom recoverable power, two pressure sensors are installed in the rodless and rod chamber of the boom cylinder in candidate excavators. Corresponding parameters are recorded as $p_{a}$ and $p_{b}$. The power analysis models $P_{L}$ and $P_{B}$ are determined based on these test parameters.

The load power demand of HEs can be obtained by the output power of the two hydraulic pumps and corresponding transmission efficiency, as shown in the following equation:

$$
P_{L}=\left(\frac{p_{1} V_{1}}{\eta_{1}}+\frac{p_{2} V_{2}}{\eta_{2}}\right) n_{E}
$$

where $V_{1}$ and $V_{2}$ denote the displacement of hydraulic Pumps 1 and 2, respectively; $\eta_{1}$ and $\eta_{2}$ represent the efficiency (including mechanical and volumetric efficiency) of the hydraulic pumps. 


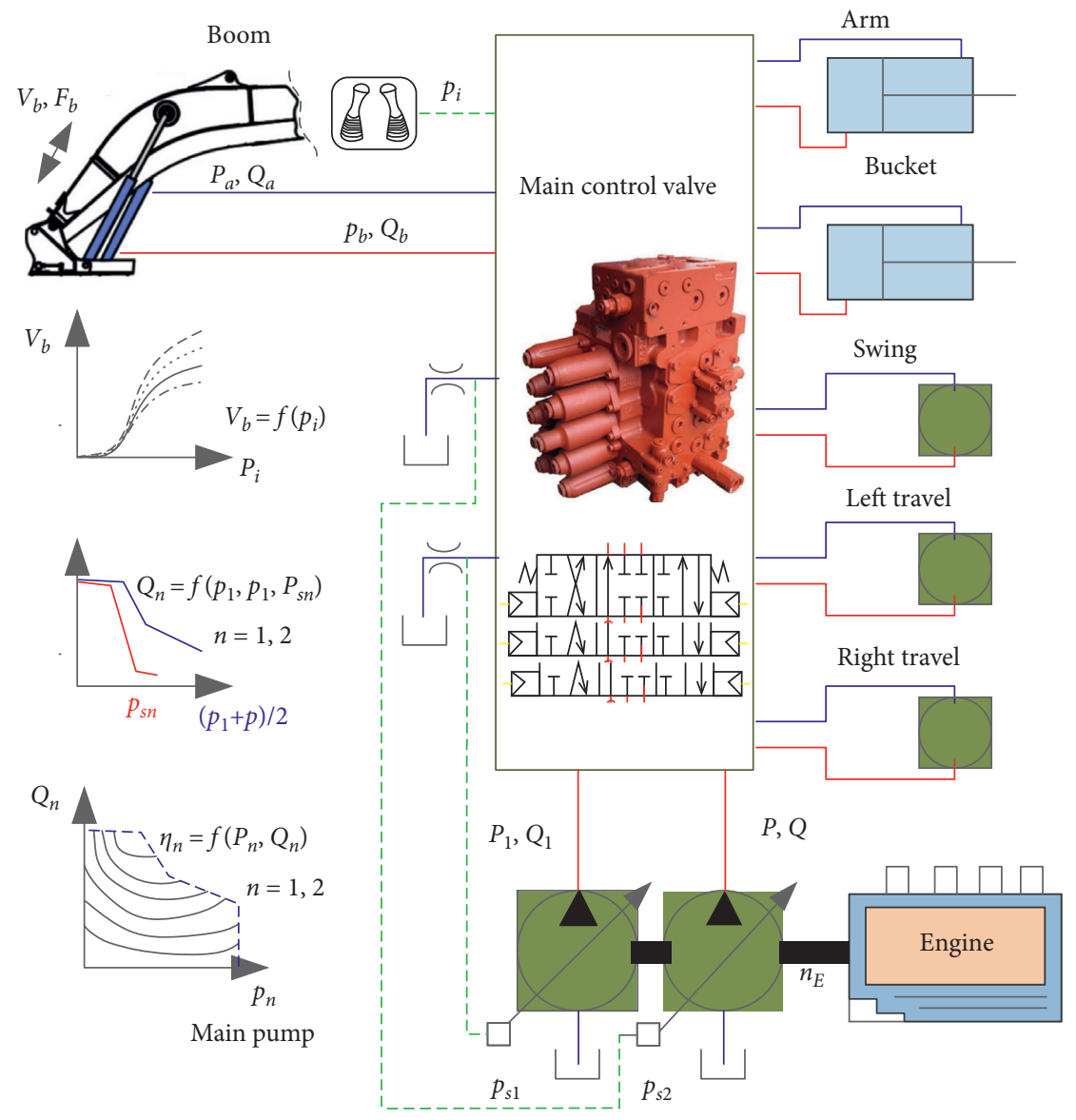

Figure 2: Principle of the SWE230B power system.

$\eta_{1}$ and $\eta_{2}$ can be obtained by discrete interpolation of threedimensional tables according to bench test data of the hydraulic pumps [27]. Displacement of the two pumps is determined by the control characteristic. There are two power control modes: approximate constant power mode and negative flow mode. In the approximate constant power mode, the pump displacement (Pump 1 and Pump 2) is adjusted according to the operating pressure average $\left(p_{d}\right)$ of Pump 1 to achieve a constant of the total input power of the dual pump. In the negative flow control mode, the pump displacement (Pump 1 or Pump 2) is reversely controlled by differential pressure. Negative flow control takes precedence over constant power control. The characteristic curves of $p_{d}, p_{s 1}$, and $p_{s 2}$ appear in Figure 3.

The curve of Figure 3 is linearly fitted as follows:

$$
\begin{aligned}
& V_{s n}= \begin{cases}V_{\max }, & \left(p_{s n} \leq 1.18\right), \\
\left(-0.5 p_{s n}+1.59\right) V_{\max }, & \left(1.18<p_{s n} \leq 2.95\right), \\
0.113 V_{\max }, & \left(p_{s n}>2.95\right),\end{cases} \\
& V_{d}= \begin{cases}\left(1-0.0019 p_{d}\right) V_{\max }, & \left(0<p_{s n} \leq 14.9\right), \\
\left(1.97-0.051 p_{d}\right) V_{\max }, & \left(14.9<p_{s n} \leq 27.4\right), \\
\left(1.16-0.0195 p_{d}\right) V_{\max }, & \left(27.4<p_{s n} \leq 34.34\right),\end{cases}
\end{aligned}
$$

where $p_{s n}$ represents $p_{s 1}$ and $p_{s 2} ; V_{s n}$ represents $V_{s 1}$ and $V_{s 2}$, which is the displacement determined by $p_{s 1}$ and $p_{s 2}$; and $V_{d}$ is the displacement determined by average pressure $p_{d}$.
According to the priority principle of displacement regulation, the displacement output model of Pump 1 and Pump 2 is determined using the following equations:

$$
\begin{aligned}
& V_{1}=\min \left\{V_{s 1}\left(p_{s 1}\right), V_{d}\left(p_{d}\right)\right\}, \\
& V_{2}=\min \left\{V_{s 2}\left(p_{s 2}\right), V_{d}\left(p_{d}\right)\right\}, \\
& p_{d}=\frac{\left(p_{1}+p_{2}\right)}{2} .
\end{aligned}
$$

Boom-lowering is a process in which gravitational potential energy is released and converted to pressure energy. The conversion rate (i.e., theoretically recoverable power) is described as follows:

$$
p_{B}=F_{B} v_{B},
$$

where $F_{B}$ is the force exerted by the boom on the piston rod of the boom cylinder and $v_{B}$ is the cylinder piston velocity. According to the force balance condition of the boom cylinder, $F_{B}$ can be calculated as follows:

$$
F_{B}=p_{b} A_{b}-p_{a} A_{a}-m \dot{v}_{b}-B v_{b},
$$

where $A_{a}$ and $A_{b}$ are the area of the rod and rodless chamber of the cylinder; $p_{a}$ and $p_{b}$ are the pressure of the rod and 


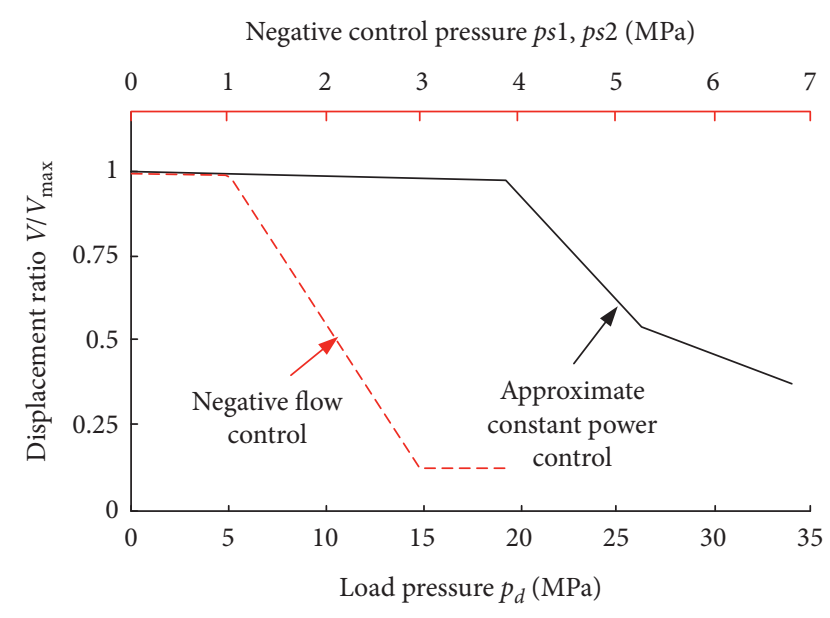

FIgURE 3: Output characteristic curve of the hydraulic pump.

rodless chamber of the cylinder; $m$ is the equivalent mass of the boom cylinder piston; and $B$ is the damping coefficient.

In conventional excavators, cylinder piston velocity $v_{b}$ is not sensed and recorded; therefore, estimating $v_{b}$ by other sensed parameters is necessary. The system principle in Figure 2 indicates that the excavator action is an open-flow velocity control loop based on hydraulic pilot. Because the boom-lowering process is mainly powered by boom gravitation, hydraulic pumps only provide low-pressure oil to prevent suction. Therefore, $v_{b}$ is mainly related to the pressure of the pilot handle $\left(p_{i}\right)$. Many data based on pilot pressure $p_{i}$ and boom velocity $v_{b}$ can thus be collected under laboratory conditions, and the approximated function relationship between the two can be fitted. The specific fitting process is described in Section 2.3.

2.3. Acquisition and Verification of Model Data. The SWE230B HE was modified in the laboratory. A velocity/ displacement sensor, pressure sensor of the rodless and rod chamber of the boom cylinder, and flow meters of the pumps were added to the conventional sensing system. Tested parameters are listed in Table 1.

Figure 4 displays a flow comparison curve of hydraulic Pump 1. The solid blue line is the measured flow rate, the red dotted line is the flow value (calculated using the flow model in Section 2.2), and the green line is the relative error of the two. The calculated value agreed well with the measured value. However, because the model neglected the dynamic response characteristics of the system, the error was large in areas where the flow rate changed rapidly, reaching $23.1 \%$. Most error was controlled within $10 \%$, and the average deviation was $5.98 \%$. This deviation is acceptable for system energy efficiency analysis.

Figure 5 illustrates the relationship between the handle pilot pressure $p_{i}$ and boom cylinder piston velocity $v_{b}$. The green crosses indicate measured data points, and the blue stars denote data from the fitting function. The fitting curve reflects the trend of the boom handle control characteristic, which was divided into an operation dead zone (0-0.7 MPa), approximate linear interval $(0.5-2.5 \mathrm{MPa})$, and operation saturation interval (2.5-3.2 MPa). The SWE230B hydraulic system is an open-loop throttling system. The hydraulic cylinder piston velocity is related to the handle pressure signal and other uncertain load disturbances (e.g., working posture). Therefore, the relationship between the measured pilot pressure $p_{i}$ and cylinder piston velocity has a certain degree of dispersion, but the fitted curve can reflect the actual velocity characteristics of the boom cylinder; thus, the curve can be used to evaluate boom energy efficiency.

\section{Microcycle Structure and Cluster Analysis Method}

3.1. Large Working Data Acquisition. To ensure that the proposed comprehensive driving cycle can reflect real-world HE characteristics, especially considering the influence of different driving habits and tasks, 10 SWE230B excavators in different parts of southern China (see Figure 6) were considered. The excavator data acquisition terminal continuously collected working data and includes built-in GPS positioning, wireless transmission, and internal memory modules. The terminal can save excavator working data in internal memory and transmits this information along with GPS coordinates to the management platform database through the wireless network during the nonworking period. To obtain sufficient sample data, we gathered working data continuously for one month. The acquisition frequency was $5 \mathrm{~Hz}$, and a total of 54,533,836 pieces of valid test data were collected. The raw data were substituted into the model in Section 2.3 to obtain data for load power demand $P_{L}$ and boom recoverable power $P_{B}$. In total, 1,500 pieces of data (300 s) were randomly intercepted as shown in Figure 7.

3.2. Microcycle Sample Partitioning Method. Figure 7 reveals that the HE working data exhibited clear periodicity, which was directly related to boom motion. Therefore, to facilitate analysis, the working data were divided into several microcycle samples using the boom motion state for categorization. Taking the boom down operation flag as a microcycle starting point, a complete microcycle structure could be obtained as shown in Figure 8 .

Considering that we mainly sought to construct a driving cycle for energy recovery analysis, and working data for low load power (e.g., idle time) and nonboom operating conditions (e.g., travel time) were deleted. Ultimately, 772,562 microcycles of valid samples were obtained and used to establish a sample database. Thirteen feature parameters were selected to describe the microcycle samples; accompanying descriptions and symbols are shown in Table 2.

Due to the sensitivity of acquisition equipment, the raw working data curve often exhibits noise interference. The Epanechnikov kernel density function can be used for filtering [28]. The basic principle of kernel function filtering is to use a certain neighborhood of the current time as the calculation area, and other data points in the neighborhood are assigned different weighting coefficients. The kernel function weighting coefficient can be determined by the following equations: 
TABLE 1: Tested parameters of laboratory excavator.

\begin{tabular}{lc}
\hline Parameters & Description \\
\hline$p_{1}$ & Pump 1 outlet pressure \\
$p_{2}$ & Pump 2 outlet pressure \\
$Q_{1}$ & Pump 1 outlet flow \\
$Q_{2}$ & Pump 2 outlet flow \\
$p_{s 1}$ & Negative flow pressure of Pump 1 \\
$p_{s 2}$ & Negative flow pressure of Pump 2 \\
$v_{b}$ & Cylinder piston velocity \\
$p_{b}$ & Rod chamber pressure of cylinder \\
$p_{a}$ & Rodless chamber pressure of cylinder \\
$n_{E}$ & Engine speed \\
\hline
\end{tabular}

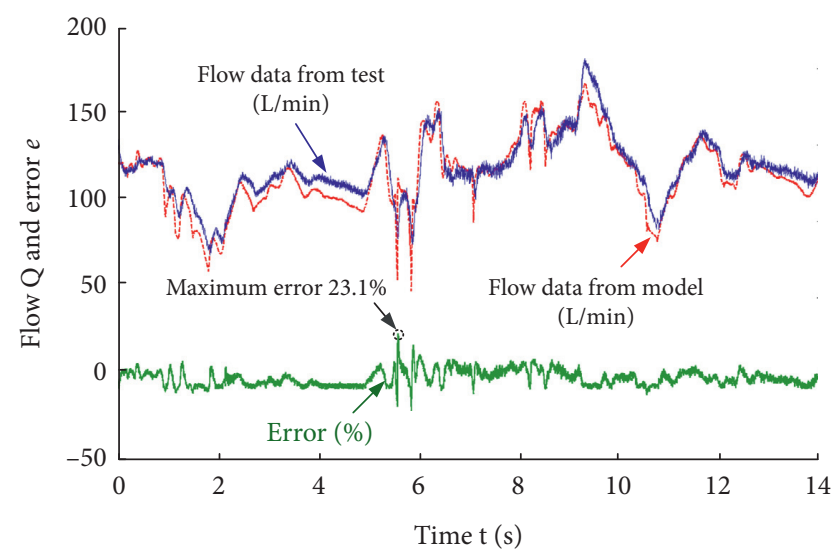

Figure 4: Flow comparison curve of hydraulic Pump 1.

$$
\begin{aligned}
\delta_{i j} & =\frac{w_{i j}}{\sum_{k=i-h}^{i+h} w_{\left(u_{i} k\right)}}, \\
W_{u_{i j}} & = \begin{cases}\frac{3}{4}\left(1-u_{i j}^{2}\right), & \left(\left|u_{i j}\right| \leq 1\right), \\
0, & \left(\left|u_{i j}\right|>1\right),\end{cases} \\
u_{i j} & =\frac{i-j}{h},
\end{aligned}
$$

where $i$ is the current time; $h$ is the neighborhood radius of current time $i$; and $j$ is the other time to be calculated in the neighborhood of $i$.

Then, the power estimate of the current time $i$ is

$$
\widehat{P}_{i}=\sum_{j=i-h}^{i+h} \delta_{i j} P_{j},
$$

where $P_{j}$ is the $j_{\text {th }}$ power value in the neighborhood $h$ (i.e., the result of filtering the kernel function).

Filtered microcycle samples were statistically analyzed using the characteristic parameters specified in Table 2, and a microcycle sample space was then constructed as shown in Table 3.
3.3. Classification Statistics Based on Working Characteristics. Due to the large number of characteristic parameters describing the microcycle, some may be linearly correlated. Principal component analysis was thus used to reduce the dimensionality of feature parameters. The microcycle sample space was transformed into the main component score space, which was used for clustering analysis [29].

Because the unit and amplitude of characteristic parameters differed, to unify the variation scale of feature parameters in the sample space, the parameters were first standardized according to the following equation:

$$
z_{j p}=\frac{x_{j p}-\overline{x_{p}}}{\sqrt{s_{p p}}}
$$

where $x_{j p}$ is the $p^{\text {th }}$ parameter value of the $j^{\text {th }}$ sample; $x_{p}$ is the statistical mean of the $p^{\text {th }}$ parameter; $s_{p p}$ is the statistical variance of the $p^{\text {th }}$ parameter; and $z_{j p}$ is the value after $x_{j p}$ is normalized.

After standardization, principal component analysis was performed using MATLAB software to obtain 13 new principal component variables. The principal component variances and their contribution rates are presented in descending order in Table 4. The cumulative contribution rate of the first five principal component variables reached $94.13 \%$, suggesting that these variables reflect most changes in the sample space. Therefore, the first five principal component variables were chosen to form the principal component score matrix, as shown in Table 5, where P1-P5 represent the selected five principal component variables.

The AK-means clustering algorithm was used to cluster the principal component score matrix in Table 5. Finally, the microcycle samples were divided into three classes. To visually examine the classification effect, sample scatter plots of the first two principal components were plotted at a point density of $1 \%$ (Figure 9). The boundary between every two classes was relatively clear; the sample points of the first two classes were highly concentrated, where as the intervals of the third type of sample points were larger and more dispersed. In the direction of the first principal component coordinate, the various center-point (gray solid circles) production intervals were larger; the second principal component coordinate direction was smaller. Numerous scatter patterns are readily distinguishable, and the classification results are ideal. We have therefore analyzed various statistical characteristics accordingly.

Table 6 shows statistical analysis results of characteristic parameters with large differences among the three sample types. $P_{s}$ denotes the proportion of each sample. The proportions of samples of Classes 1 and 2 were $61.31 \%$ and $27.40 \%$, respectively. Both were dominant, and the sum of ratios was close to $90 \%$, indicating that Classes 1 and 2 represented the main operating conditions. Class 3 only accounted for $11.29 \%$ of all samples, indicating a secondary operating condition.

In Class 1, the microcycle duration, maximum load demand power, and maximum boom recoverable power were highest; thus, Class 1 embodied a working state of maximum excavation resistance, large boom movement, and 


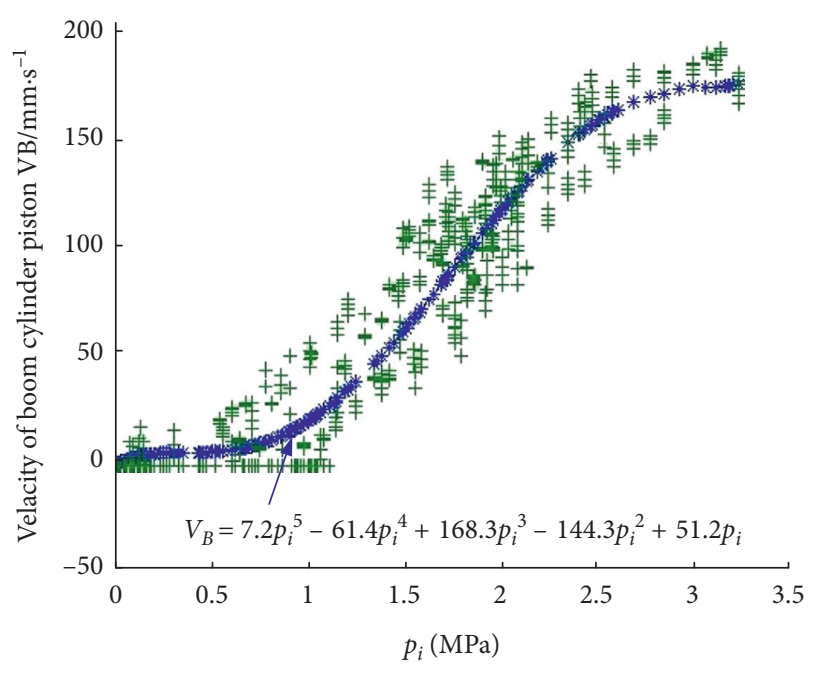

+ Velocity data from test

* Data from fitting function

FIGURE 5: Relationship between handle pressure and boom cylinder piston velocity.

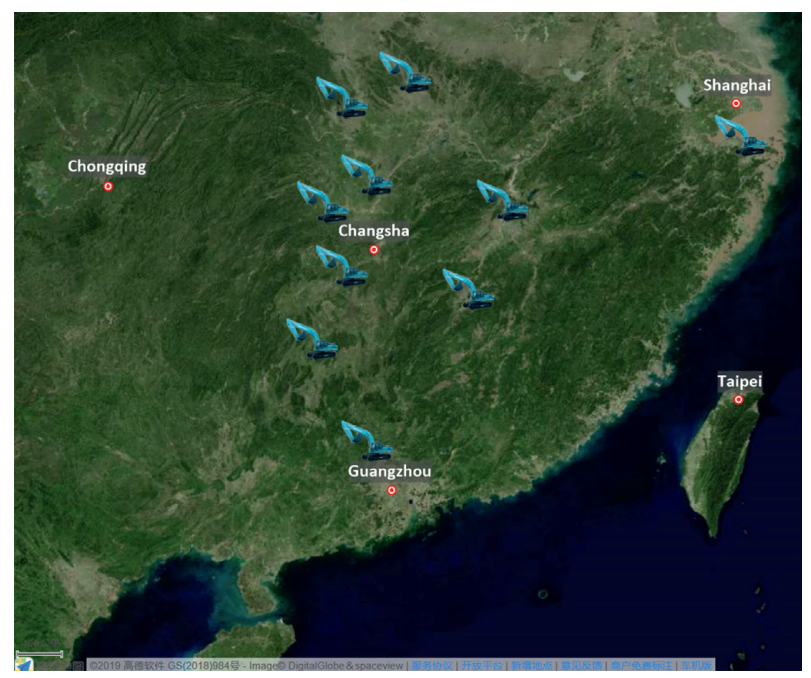

FIgURE 6: Geographical distribution of selected excavator.

high velocity. Class 1 therefore reflected heavy-duty conditions (e.g., rapid excavation and loading). In Class 3, the microcycle duration was minimal, indicating that the movement amplitude of actuators was small. The average load demand power was also small, suggesting that the hydraulic system was in a minimum power mode; this represents a fine working condition (e.g., slope trimming and earth grading). Most parameters in Class 2 were between those in Classes 1 and 3, such as microcycle duration, average load demand power, and boom recyclable power. Class 2 thus coincided with general earth-moving (e.g., trenching and filling).

To further estimate the energy-saving potential of energy recovery under the three identified working conditions, the following assumptions were made:
(1) Recoverable power utilization reached 100\% (ideal)

(2) Boom recoverable energy was directly applied and used to reduce power output of the original prime mover system

(3) The recovered energy did not affect the transmission efficiency of the prime mover (i.e., the efficiency did not become better or worse)

Taking the average boom recoverable power as the ratio of average load demand power per Table 6, the theoretical energy recovery efficiency of Classes 1,2 , and 3 was $17.45 \%$, $13.60 \%$, and $11.88 \%$, respectively. Combined with characteristics of the three classes, the energy-saving efficiency during the rapid excavation and loading process (Class 1) was highest, where as that of Class 3 was lowest (e.g., under fine operating conditions). Taking the proportion Ps of the three classes in the sample as the weighting coefficient, the energy-saving potential of the boom energy recovery in the whole sample space was $15.76 \%$.

\section{Construction of Comprehensive Driving Cycle Based on Markov Chain}

4.1. Markov Chain. The Markov chain is a typical stochastic process, with the characteristics of "future" and "past" being independent under the condition of "now"; that is, the state value at time $t+1$ is only related to the current time $t$ and has nothing to do with time $t-1$; this property is called "no aftereffect." Many researchers have used the Markov chain to construct (i.e., predict) vehicle driving cycles [30-32]. Given the complexity of the working environment and personal driving habits, the working state of excavators is highly random. Considering that the excavator is a task-oriented work machine, it also shows no obvious aftereffects. For example, the lowering height of the excavator boom is generally only related to that at the previous moment (i.e., the height of the soil that has been excavated), regardless of the previous history. Therefore, the Markov chain can be used to construct the excavator driving cycle. However, due to the diversity of excavator working conditions, it is difficult to construct a driving cycle that reflects real-world scenarios. Based on the classification of numerous working data, we constructed the driving cycle for three types of working conditions.

The basic method of building a driving cycle based on a Markov chain is to consider the time series of working data as a complete random process $[33,34]$. Working data can be divided into sections, each of which represents a different working state; then, the time series of working data can be converted into a time series of the working state. The next state is only related to the current state. The transition probability between these two states can be depicted by a state transition probability rectangle, as shown in equation (11). With this rectangle, the program can randomly generate a random sequence of states. Finally, the working state sequence is inverted into working data that meet the characteristics of the sample space. 


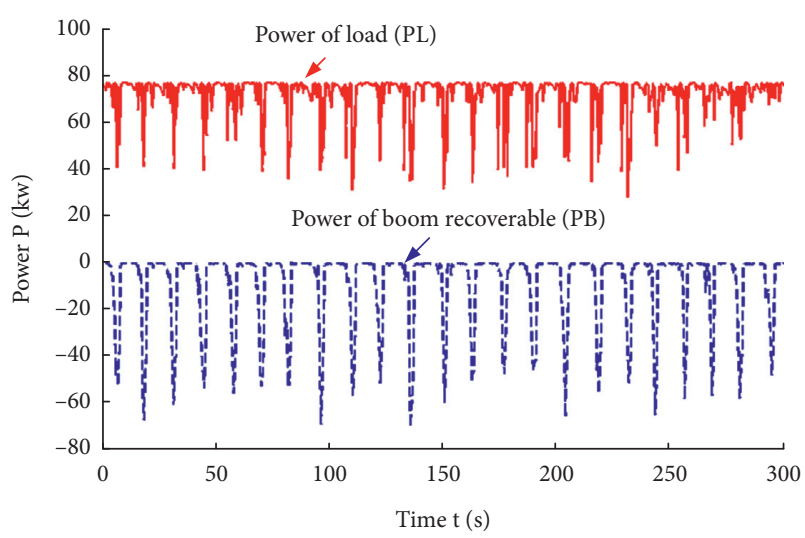

Figure 7: 1,500 pieces of power data.

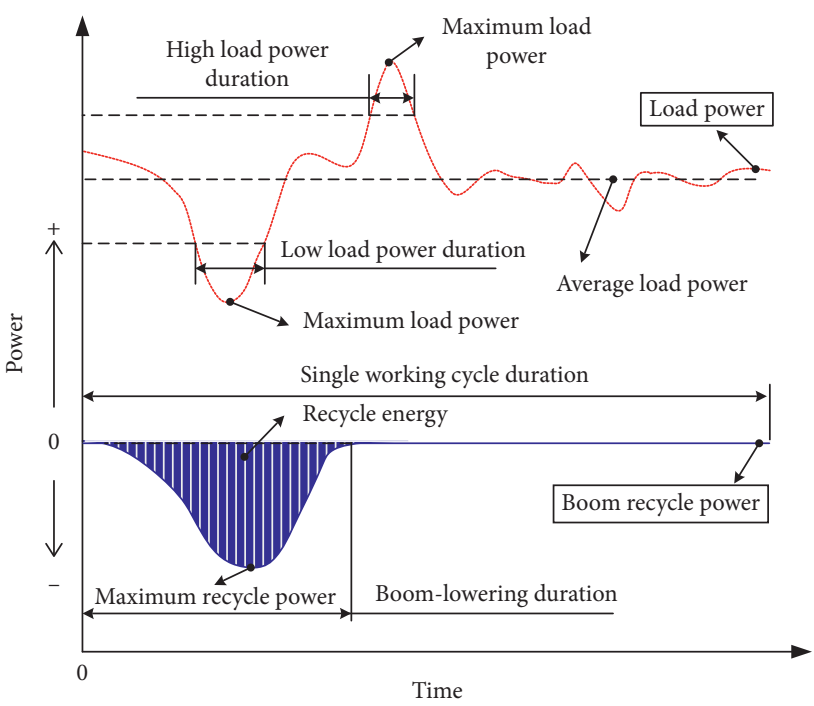

FIGURE 8: Microcycle structure of hydraulic excavator.

$$
\mathbf{S}=\left(S_{i j}\right)_{M \times M}=\left[\begin{array}{cccc}
S_{11} & S_{12} & \ldots & S_{1 M} \\
S_{21} & S_{22} & \ldots & S_{2 M} \\
\vdots & \vdots & \ddots & \vdots \\
S_{M 1} & S_{M 2} & \ldots & S_{M M}
\end{array}\right],
$$

where $M$ is the number of states and $S_{i j}$ is the probability of transitioning from the current state $i$ to the next state $j$.

The states described herein include two parameters, load demand power and boom recoverable power. These parameters are related but relatively independent; therefore, the state must be determined based on a combination of these two parameters. First, the load demand power and boom recoverable power are divided into several intervals according to the uniform power interval. The specified power interval span in this paper was $15 \mathrm{~kW}$, and the start was $0 \mathrm{~kW}$. The load demand power in $[0,15)$ was taken as the substate $P_{L}(1)$. That, in interval $[15,30)$, was taken as the substate $P_{L}(2)$ and so on. With a maximum power of $90 \mathrm{~kW}$ as the upper limit, a total of six substates were produced.
TABLE 2: Microcycle feature parameters.

\begin{tabular}{lc}
\hline Parameters/unit & Description \\
\hline$(T / s)$ & Microcycle duration \\
$\left(T_{b} / s\right)$ & Boom-lowering duration \\
$\left(P_{L_{-} \text {max }} / \mathrm{kW}\right)$ & Average load demand power \\
$\left(P_{L_{-} \text {min }} / \mathrm{kW}\right)$ & Minimum load demand power \\
$\left(P_{L_{-} s d} / \mathrm{kW}\right)$ & Standard deviation of load demand power \\
$\left(E_{L} / \mathrm{kWh}\right)$ & Load demand energy during a microcycle \\
$\left(P_{B_{-} \text {max }} / \mathrm{kW}\right)$ & Maximum boom recoverable power \\
$\left(P_{B_{-} \text {avg }} / \mathrm{kW}\right)$ & Average boom recoverable power \\
$\left(P_{B_{-} s d} / \mathrm{kW}\right)$ & Standard deviation of boom recoverable power \\
$\left(E_{B} / \mathrm{kWh}\right)$ & Boom recoverable energy during a microcycle \\
$\left(r_{B L} / \%\right)$ & Ratio between boom PB and PL \\
$\left(P_{\text {Lavg_down }} / \mathrm{kW}\right)$ & Average load demand power during boom- \\
\hline
\end{tabular}

TABLE 3: Microcycle feature parameters.

\begin{tabular}{lccccc}
\hline No. & $T$ & $T_{b}$ & $\ldots$ & $r_{B L}$ & $P_{\text {Lavg_down }}$ \\
\hline 1 & 13.5 & 3.8 & $\ldots$ & 17.3 & 57.2 \\
2 & 13.1 & 3.7 & $\ldots$ & 15.9 & 57.1 \\
$\vdots$ & $\vdots$ & $\vdots$ & $\vdots$ & $\vdots$ & \\
772561 & 11.7 & 3.5 & $\ldots$ & 19.2 & 59.1 \\
772562 & 12.2 & 3.5 & $\ldots$ & 18.3 & 58.6 \\
\hline
\end{tabular}

Similarly, the boom recoverable power began from 0 with an interval of $15 \mathrm{~kW}$. That, in $(-15,0]$, was taken as the substate $P_{B}(1)$, with substate $P_{B}(2)$ in interval $(-30,15)$, and so on. A total of six substates were thus obtained. Finally, two substate sets $P_{L}$ and $P_{B}$ were combined; specifically, $P_{L}(1)$ and $P_{B}(1)$ were combined into State $1 ; P_{L}(1)$ and $P_{B}(2)$ were combined into State 2; and so on. $P_{L}(6)$ and $P_{B}(6)$ were combined into State 36 . Considering the large sample size in this study, maximum likelihood estimation was used to obtain the state transition probability. This approach can allow the statistical result to better approximate the actual situation and reduce the required calculation as much as possible. The corresponding state transition probability equation is as follows:

$$
S_{i j}=\frac{N_{i j}}{\sum_{j} N_{i j}},
$$

where $N_{i j}$ represents the transition frequency from state $i$ to state $j$ and $\sum_{j} N_{i j}$ represents the frequency from state $i$ to either state. Considering that a certain correlation exists between $P_{L}$ and $P_{B}$, an invalid combination may arise in which the combination of $\sum_{j} N_{i j}$ equals 0 . In the following specific analysis, this invalid combination was removed, and the subsequent state number was shifted forward to maintain state continuity; as such, the actual total number of states may be less than 36 .

According to the classification results in Section 3, the state transition probability matrix can be calculated separately. Figure 10 is a state transition probability matrix diagram of Class 1 . Table 7 shows the original data used in Figure 10. Vertical and horizontal represent the current state 
TABLE 4: Principal component significance assessment.

\begin{tabular}{lcc}
\hline Principal component & Variance & Contribution rate (\%) \\
\hline 1 & 3.5370 & 38.31 \\
2 & 3.0431 & 32.96 \\
3 & 1.2686 & 13.74 \\
4 & 0.5661 & 6.13 \\
5 & 0.2761 & 2.99 \\
6 & 0.1293 & 1.40 \\
7 & 0.1090 & 1.18 \\
8 & 0.0970 & 1.05 \\
9 & 0.0818 & 0.89 \\
10 & 0.0499 & 0.54 \\
11 & 0.0375 & 0.41 \\
12 & 0.0208 & 0.23 \\
13 & 0.0156 & 0.17 \\
\hline
\end{tabular}

TABle 5: Principal component score matrix.

\begin{tabular}{lccccc}
\hline No. & P1 & P2 & P3 & P4 & P5 \\
\hline 1 & -2.7001 & -1.4604 & -0.7007 & 0.3049 & 0.6539 \\
2 & 0.5756 & -0.1378 & -0.1138 & -0.1925 & $\vdots$ \\
$\vdots$ & $\vdots$ & $\vdots$ & $\vdots$ & -0.6657 \\
772561 & 1.7659 & 1.2944 & -0.1906 & -0.3453 & 0.0868 \\
772562 & 1.5938 & 1.0047 & -0.0911 & -0.5186 \\
\hline
\end{tabular}

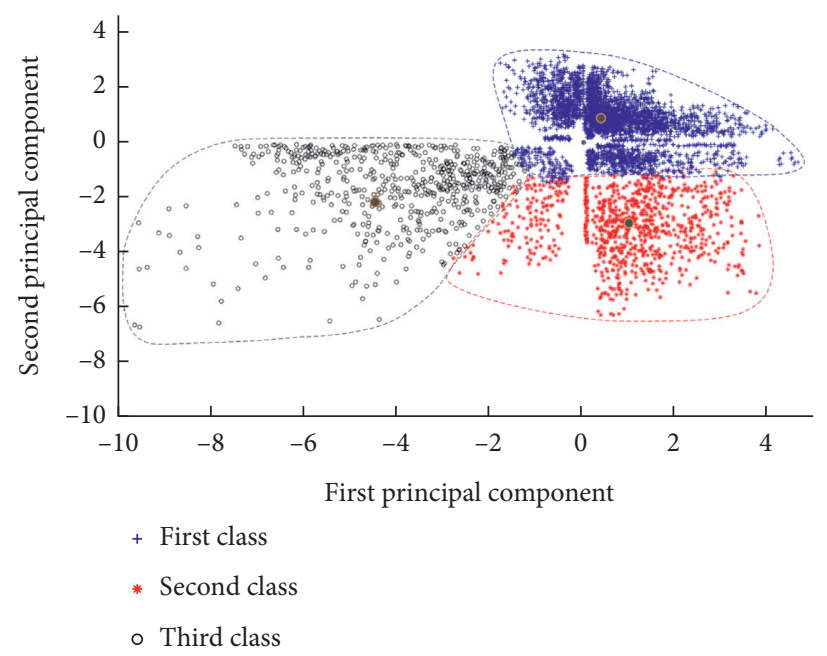

FIgURE 9: Results of cluster analysis.

TABle 6: Principal component score matrix.

\begin{tabular}{lccc}
\hline Characteristic parameters & Class 1 & Class 2 & Class 3 \\
\hline$\left(P_{s} / \%\right)$ & 61.31 & 27.40 & 11.29 \\
$(T / s)$ & 14.36 & 12.09 & 10.28 \\
$\left(P_{L_{-} \text {max }} / \mathrm{kW}\right)$ & 89.04 & 82.05 & 80.91 \\
$\left(P_{L m d r} / \mathrm{kW}\right)$ & 63.82 & 59.70 & 56.83 \\
$\left(P_{L_{-} s d} / \mathrm{kW}\right)$ & 10.63 & 8.57 & 7.22 \\
$\left(P_{B_{-} \max } / \mathrm{kW}\right)$ & 81.78 & 50.19 & 45.90 \\
$\left(P_{\mathrm{Bmdr}} / \mathrm{kW}\right)$ & 11.14 & 8.12 & 6.75 \\
$\left(P_{B_{-} s d} / \mathrm{kW}\right)$ & 17.69 & 12.50 & 10.11 \\
\hline
\end{tabular}




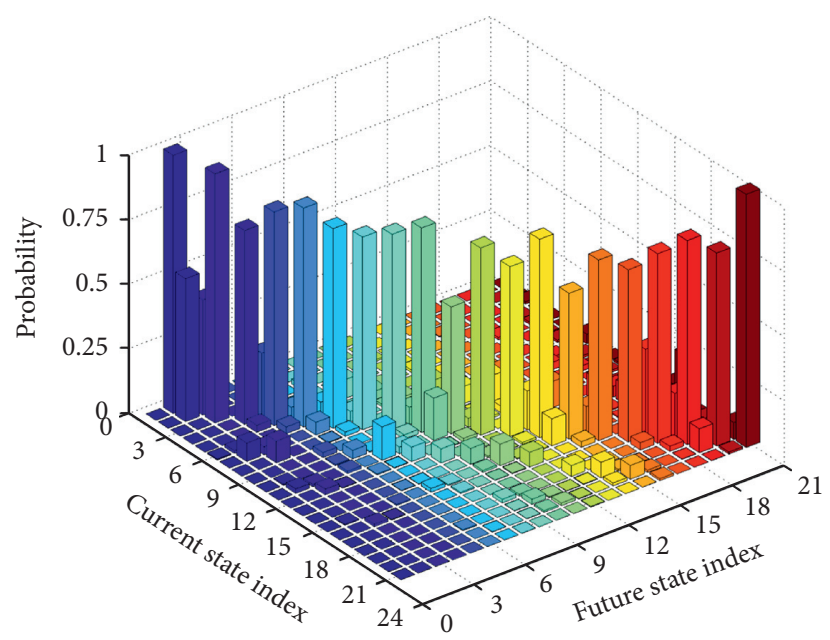

FIGURE 10: Markov matrix of Class 1.

and future state, respectively. The total state number of Class 1 after removing invalid states was 21 . The maximum probability values are on the diagonal line, suggesting that the excavator tended to maintain a current power interval. The probability value was smallest in the state away from the diagonal, indicating that the power variation rarely fluctuated greatly. These results are consistent with the sample data characteristics. For Classes 2 and 3, the same approach was used to produce the Markov transition probability matrix.

4.2. Comprehensive Driving Cycle. Combined with the state transition probability matrix, MATLAB was used to produce the driving cycle class by class. The specific implementation steps are as follows:

(1) Generate a uniformly distributed random number $r$ in the $(0,1)$ interval and set the current state $i$ to 1 . The next state $j$ is determined according to equation (13). Then, repeat the above calculation process for the next state, after which a sequence of states is obtained; this sequence must satisfy the corresponding time length that is not less than the minimum duration of the class, after which the last state returns to initial State 1 again.

$$
j=\underset{k \in[1, M]}{\operatorname{argmin}}\left(k \mid \sum_{g=1}^{k} S_{i g} \geq r\right) .
$$

(2) Convert the state sequence into a candidate sequence, calculated as

$$
P_{s}=[(s-1)+r] \Delta d,
$$

where $P_{s}$ represents the power value corresponding to state $s ; r$ is a random number uniformly distributed in the interval [ 01 ); and $\Delta d$ is the length of the power interval corresponding to the state.

(3) Calculate the average absolute deviation between the candidate sequence and the sample database according to the characteristic parameters of Table 6; the deviation is within $5 \%$, and it is regarded as a qualified sequence; otherwise, return to Step 1. The formula for calculating the average absolute deviation is as follows:

$$
w=\frac{\sum_{m=1}^{n}\left|C_{m}-G_{m}\right|}{n},
$$

where $n$ is the number of characteristic parameters; $C_{m}$ and $G_{m}$ are the $m^{\text {th }}$ characteristic parameter in the candidate sequence and sample database, respectively; and $w$ is the calculated average absolute deviation value.

The qualified sequence of three classes can then be combined according to the time ratio $P_{s}$ in Table 6. A comprehensive driving cycle with a total time of $68 \mathrm{~s}$ is shown in Figure 11.

Table 8 presents a statistical characteristics comparison between the constructed comprehensive driving cycle and sample database. Most deviations were small (i.e., within 5\%). The deviations in maximum load demand power and maximum boom recoverable power were relatively large, reaching 7\%-8\%. The overall deviation was within $10 \%$ with an average deviation of $4.26 \%$. These statistical results suggest that the constructed driving cycle reflected the general characteristics of comprehensive real-world working conditions. 
TABLE 7: State transition probability database of Class 1.

\begin{tabular}{cccccccccccccccccccccccc}
\hline No. & 1 & 2 & 3 & 4 & 5 & 6 & 7 & 8 & 9 & 10 & 11 & 12 & 13 & 14 & 15 & 16 & 17 & 18 & 19 & 20 & 21 \\
\hline 1 & 0.98 & 0 & 0 & 0 & 0 & 0 & 0 & 0.03 & 0 & 0 & 0 & 0.02 & 0 & 0 & 0 & 0 & 0 & 0 & 0 & 0 & 0 \\
2 & 0 & 0.53 & 0 & 0.02 & 0 & 0 & 0 & 0 & 0 & 0 & 0 & 0 & 0 & 0 & 0 & 0.13 & 0 & 0 & 0 & 0.01 & 0 \\
3 & 0 & 0 & 0.92 & 0 & 0 & 0 & 0 & 0 & 0 & 0.01 & 0 & 0 & 0 & 0 & 0 & 0 & 0 & 0.02 & 0 & 0 & 0 \\
4 & 0 & 0 & 0 & 0.72 & 0.03 & 0 & 0 & 0 & 0 & 0 & 0 & 0 & 0 & 0.03 & 0 & 0 & 0 & 0 & 0 & 0 & 0 \\
5 & 0 & 0 & 0 & 0.04 & 0.78 & 0.05 & 0 & 0 & 0 & 0 & 0 & 0 & 0 & 0 & 0 & 0 & 0.02 & 0 & 0 & 0 & 0.01 \\
6 & 0 & 0 & 0 & 0 & 0.04 & 0.77 & 0.12 & 0 & 0 & 0 & 0 & 0 & 0 & 0 & 0 & 0 & 0 & 0 & 0.01 & 0 & 0 \\
7 & 0 & 0.1 & 0 & 0 & 0 & 0.07 & 0.71 & 0.06 & 0 & 0 & 0 & 0 & 0 & 0 & 0.01 & 0 & 0 & 0.15 & 0 & 0 & 0 \\
8 & 0 & 0 & 0.13 & 0 & 0 & 0 & 0.02 & 0.76 & 0.07 & 0 & 0 & 0 & 0.02 & 0 & 0 & 0 & 0 & 0 & 0 & 0.03 & 0.02 \\
9 & 0 & 0 & 0 & 0 & 0.02 & 0 & 0 & 0 & 0.82 & 0.11 & 0 & 0 & 0 & 0 & 0 & 0.03 & 0 & 0 & 0 & 0 & 0 \\
10 & 0 & 0 & 0 & 0 & 0.01 & 0.03 & 0 & 0 & 0 & 0.88 & 0.09 & 0 & 0 & 0 & 0 & 0 & 0 & 0 & 0 & 0 & 0 \\
11 & 0 & 0.02 & 0.01 & 0 & 0.01 & 0 & 0.13 & 0 & 0 & 0.2 & 0.56 & 0.11 & 0 & 0 & 0.01 & 0 & 0 & 0 & 0.16 & 0 & 0 \\
12 & 0 & 0 & 0.03 & 0 & 0 & 0 & 0 & 0.07 & 0 & 0 & 0.03 & 0.86 & 0.08 & 0 & 0 & 0 & 0 & 0 & 0 & 0 & 0 \\
13 & 0 & 0 & 0 & 0 & 0 & 0.01 & 0 & 0 & 0.07 & 0 & 0 & 0 & 0.79 & 0.12 & 0 & 0 & 0.07 & 0 & 0 & 0 & 0.01 \\
14 & 0 & 0 & 0 & 0 & 0 & 0 & 0 & 0 & 0 & 0.09 & 0 & 0 & 0 & 0.87 & 0.11 & 0 & 0 & 0 & 0 & 0 & 0 \\
15 & 0 & 0 & 0 & 0 & 0 & 0 & 0.02 & 0 & 0 & 0 & 0.08 & 0.01 & 0 & 0.12 & 0.62 & 0.1 & 0 & 0 & 0 & 0 & 0 \\
16 & 0 & 0 & 0.02 & 0 & 0 & 0 & 0 & 0.02 & 0 & 0.01 & 0.02 & 0.08 & 0 & 0 & 0.03 & 0.8 & 0.06 & 0 & 0 & 0 & 0 \\
17 & 0 & 0 & 0 & 0 & 0 & 0 & 0 & 0.01 & 0 & 0 & 0.02 & 0 & 0.01 & 0 & 0 & 0.01 & 0.79 & 0.14 & 0.06 & 0.02 & 0 \\
18 & 0 & 0 & 0 & 0 & 0 & 0 & 0.01 & 0 & 0 & 0 & 0 & 0 & 0.04 & 0.01 & 0 & 0 & 0.01 & 0.81 & 0.12 & 0 & 0 \\
19 & 0 & 0 & 0 & 0 & 0 & 0 & 0.01 & 0 & 0 & 0.04 & 0.01 & 0 & 0.01 & 0.09 & 0.01 & 0 & 0 & 0.03 & 0.8 & 0 & 0 \\
20 & 0 & 0 & 0 & 0 & 0 & 0 & 0 & 0 & 0.01 & 0.03 & 0 & 0.01 & 0 & 0.02 & 0.06 & 0.01 & 0 & 0.02 & 0.08 & 0.78 & 0 \\
21 & 0 & 0 & 0 & 0 & 0 & 0 & 0 & 0 & 0 & 0.01 & 0 & 0 & 0.01 & 0 & 0 & 0.01 & 0 & 0 & 0 & 0.01 & 0.96 \\
\hline
\end{tabular}

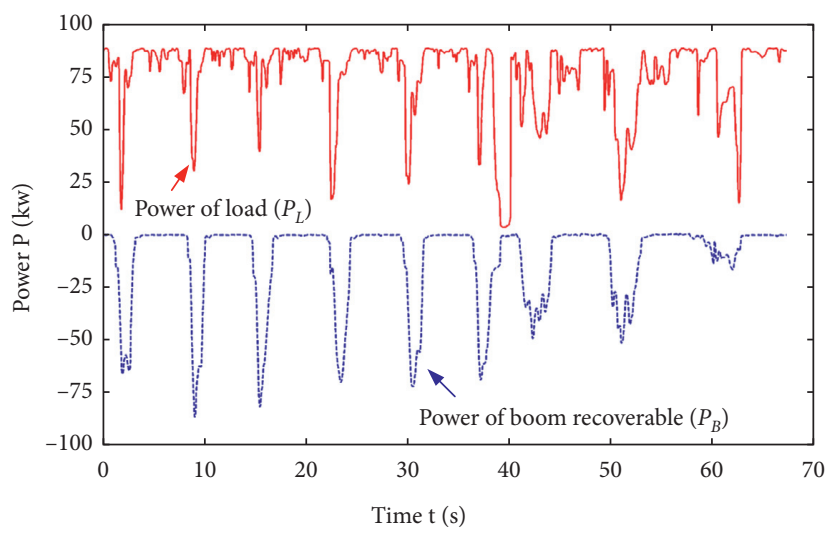

FIGURE 11: Comprehensive driving cycle of hydraulic excavator.

TABLE 8: Comparison of comprehensive driving cycle and sample database.

\begin{tabular}{lccc}
\hline Parameters & $\begin{array}{c}\text { Sample } \\
\text { database }\end{array}$ & Comprehensive cycle & Deviation/\% \\
\hline$(T / s)$ & 13.23 & 12.93 & 2.31 \\
$\left(P_{L_{-} \text {max }} / \mathrm{kW}\right)$ & 85.93 & 92.64 & 7.81 \\
$\left(P_{L m d r} / \mathrm{kW}\right)$ & 61.70 & 64.51 & 4.55 \\
$\left(P_{L_{-} s d} / \mathrm{kW}\right)$ & 9.65 & 9.98 & 3.40 \\
$\left(P_{B_{-} \text {max }} / \mathrm{kW}\right)$ & 68.82 & 74.06 & 7.61 \\
$\left(P_{\mathrm{Bmdr}} / \mathrm{kW}\right)$ & 9.78 & 9.46 & 3.29 \\
$\left(P_{B_{-} s d} / \mathrm{kW}\right)$ & 15.36 & 15.23 & 0.86 \\
\hline
\end{tabular}

\section{Conclusion}

In this study, we performed an energy efficiency assessment of new energy-saving products characterized by energy recovery technology. Based on a large amount of real-world working data from medium-sized HEs, we proposed a method for constructing a comprehensive driving cycle of engineering machinery. The main contributions are as follows:

(1) For the first time, a microcycle structure with demand and recoverable power parameters was proposed, and corresponding characteristic parameters were defined.

(2) Three classes of typical working conditions were obtained via principal component analysis and cluster analysis; then, the standardization of multifunctional driving cycle of a real-world excavator is realized.

(3) According to the cluster analysis results, the energysaving potential based on boom energy recovery was $17.45 \%, 13.60 \%$, and $11.88 \%$ class by class. The total energy-saving potential was $15.76 \%$. 
(4) Based on the Markov chain, a comprehensive driving cycle was constructed, the average deviation of which was $4.26 \%$. The comprehensive driving cycles can fairly reflect the general characteristics of real-world working conditions. The proposed construction method of comprehensive driving cycles based on sample space is therefore reliable and holds great promise for evaluating the energy-saving efficiency of new energy-saving concept systems.

(5) Due to the typical and general nature of the defined driving cycle parameters, the presented construction method can easily be adapted for the application on different types of mobile machinery. This could be beneficial during the development of energy-saving construction machinery, thus making testing easier and development cycle shorter.

\section{Data Availability}

The data used to support the findings of this study are available from the corresponding author upon request.

\section{Conflicts of Interest}

The author(s) declare that they have no conflicts of interest regarding the publication of this paper.

\section{Acknowledgments}

This research was supported by Hunan Provincial Natural Science Foundation (2020JJ5173), The National Natural Science Foundation of China (51809091), Hunan Provincial Science and Technology Tackling Key Project (2019GK4014), Open Foundation of the State Key Laboratory of Fluid Power and Mechatronic Systems (GZKF201813), Xiangtan Science and Technology Special Project (CXY-ZD20172001), and National Key R\&D Project (2019YFC1511500).

\section{References}

[1] M. S. Kumar and S. T. Revankar, "Development scheme and key technology of an electric vehicle: an overview," Renewable and Sustainable Energy Reviews, vol. 70, pp. 1266-1285, 2017.

[2] Y. Hao, L. Quan, H. Cheng, L. Xia, L. Ge, and B. Zhao, "Potential energy directly conversion and utilization methods used for heavy duty lifting machinery," Energy, vol. 155, pp. 242-251, 2018.

[3] A. Kumar, K. Dasgupta, and J. Das, "Achieving constant speed of a hydrostatic drive using controlled operation of the pump and enhancing its energy efficiency," ISA Transactions, vol. 90, pp. 189-201, 2019.

[4] J. Das, S. K. Mishra, A. Kumar, N. Kumar, and K. Sujit, "Study of energy recycling in electro-hydraulic system," Applied Mechanics and Materials, vol. 592-594, pp. 2244-2248, 2014.

[5] T. Lin, Q. Chen, H. Ren, W. Huang, Q. Chen, and S. Fu, "Review of boom potential energy regeneration technology for hydraulic construction machinery," Renewable and Sustainable Energy Reviews, vol. 79, pp. 358-371, 2017.

[6] L. Ge, Z. Dong, L. Quan, and Y. Li, "Potential energy regeneration method and its engineering applications in large- scale excavators," Energy Conversion and Management, vol. 195, pp. 1309-1318, 2019.

[7] J. Gong, D. Zhang, C. Liu, Y. Zhao, P. Hu, and W. Quan, "Optimization of electro-hydraulic energy-savings in mobile machinery," Automation in Construction, vol. 98, pp. 132-145, 2019.

[8] G. Jun, Z. Daqing, G. Yong et al., "Potential energy recovery method based on alternate recovery and utilization of multiple hydraulic cylinders," Automation in Construction, vol. 112, Article ID 103105, 2020.

[9] M. André, R. Joumard, R. Vidon, P. Tassel, and P. Perret, "Real-world European driving cycles, for measuring pollutant emissions from high- and low-powered cars," Atmospheric Environment, vol. 40, no. 31, pp. 5944-5953, 2006.

[10] K. Ça ğatay Bayindir, M. A. Gözüküçük, and A. Teke, “A comprehensive overview of hybrid electric vehicle: powertrain configurations, powertrain control techniques and electronic control units," Energy Conversion and Management, vol. 52, no. 2, pp. 1305-1313, 2011.

[11] H. Gong, Y. Zou, Q. Yang, J. Fan, F. Sun, and D. Goehlich, "Generation of a driving cycle for battery electric vehicles: A case study of Beijing," Energy, vol. 150, pp. 901-912, 2018.

[12] J. Lin and D. A. Niemeier, "Regional driving characteristics, regional driving cycles," Transportation Research Part D: Transport and Environment, vol. 8, no. 5, pp. 361-381, 2003.

[13] P. Seers, G. Nachin, and M. Glaus, "Development of two driving cycles for utility vehicles," Transportation Research Part D: Transport and Environment, vol. 41, pp. 377-385, 2015.

[14] J. Lin and D. A. Niemeier, "An exploratory analysis comparing a stochastic driving cycle to California's regulatory cycle," Atmospheric Environment, vol. 36, no. 38, pp. 5759-5770, 2002.

[15] IE moving machinery, Earth-moving machinery-sustainability-part 3: used machines, ISO 10987-3:2017. https://www.iso.org/standard/67163.html.

[16] Japan Construction Mechanization Association, "Earthmoving machinery-test methods for energy consumptionhydraulic excavators," JCMAS, Tokyo, Japan, 2010, http:// www.jcmanet.or.jp/jcmas/pdf/H020_2010.

[17] G 7586-2008, Hydraulic excavators-test methods.http:// www.gb688.cn/bzgk/gb/newGbInfo? $\mathrm{h}$ cno=C588AE0CC3A97FB.

[18] Y. Yin, G. Y. Grondin, K. H. Obaia, and A. E. Elwi, "Fatigue life prediction of heavy mining equipment. Part 1: fatigue load assessment and crack growth rate tests," Journal of Constructional Steel Research, vol. 63, no. 11, pp. 1494-1505, 2007.

[19] Q. Xiang, P. Lu, B. Wang, H. Zhang, and L. Xue, "Load spectrum test method of hydraulic excavator working device," China Highway and Transportation, vol. 30, no. 09, pp. 151158, 2017.

[20] X. Zhang and X. Zhai, "Segmented extrapolation of hydraulic pump load spectrum based on whole-machine test of an excavator," Mechanical Science and Technology for Aerospace Engineering, vol. 37, no. 09, pp. 1319-1324, 2018.

[21] D. Arsić, N. Gnjatović, S. Sedmak, A. Arsić, and M. Uhričik, "Integrity assessment and determination of residual fatigue life of vital parts of bucket-wheel excavator operating under dynamic loads," Engineering Failure Analysis, vol. 105, pp. 43-60, 2019.

[22] S. Zarotti, R. Paoluzzi, and G. Ganassi, "Analysis of hydraulic excavator working cycle," in Proceedings of the 11th European Regional Conference of the International Society for TerrainVehicle Systems, Bremen, Germany, October 2009. 
[23] J. Yoon, J. Kim, J. Seo, and S. Suh, "Spatial factors affecting the loading efficiency of excavators," Automation in Construction, vol. 48, pp. 97-106, 2014.

[24] J. Kim and S. Chi, "Action recognition of earthmoving excavators based on sequential pattern analysis of visual features and operation cycles," Automation in Construction, vol. 104, pp. 255-264, 2019.

[25] J. Gong, D. Zhang, y. Guo et al., "Power control strategy and performance evaluation of a novel electro-hydraulic energysaving system," Applied Energy, vol. 233-234, pp. 724-734, 2019.

[26] NN, Sunward SWE230b Medium Hydraulic Excavator Brochure (2017).http://en.sunward.com.cn/product/productdetail-2338.htm.

[27] NN, Kawasaki K3vl Axial piston pumps for open circuits in mobile, in Industrial and Marine Applications; Datasheet: Kawasaki. Japan (2014).

[28] J. Brady and M. O’Mahony, "Development of a driving cycle to evaluate the energy economy of electric vehicles in urban areas," Applied Energy, vol. 177, pp. 165-178, 2016.

[29] Q. Cao, J. Li, Y. Liu, and D. Qu, "Construction of driving cycle based on Markov chain for passenger car in Changchun city," Journal of Jilin University Engineering and Technology Edition, vol. 48, no. 5, pp. 1366-1373, 2018.

[30] H. Hemi, J. Ghouili, and A. Cheriti, "Combination of Markov chain and optimal control solved by Pontryagin's Minimum Principle for a fuel cell/supercapacitor vehicle," Energy Conversion and Management, vol. 91, pp. 387-393, 2015.

[31] H. Liu, X. Li, W. Wang, L. Han, and C. Xiang, "Markov velocity predictor and radial basis function neural networkbased real-time energy management strategy for plug-in hybrid electric vehicles," Energy, vol. 152, pp. 427-444, 2018.

[32] R. Ma, X. He, Y. Zheng, B. Zhou, S. Lu, and Y. Wu, "Realworld driving cycles and energy consumption informed by large-sized vehicle trajectory data," Journal of Cleaner Production, vol. 223, pp. 564-574, 2019.

[33] Z. Wang, J. Zhang, P. Liu, C. Qu, and X. Li, "Driving cycle construction for electric vehicles based on Markov chain and Monte Carlo method: a case study in Beijing," Energy Procedia, vol. 158, pp. 2494-2499, 2019.

[34] J. Zhang, Z. Wang, P. Liu, Z. Zhang, X. Li, and C. Qu, “Driving cycles construction for electric vehicles considering road environment: a case study in Beijing," Applied Energy, vol. 253, p. 113514, 2019. 